\title{
Pre-treatment plasma proteomic markers associated with survival in oesophageal cancer
}

\author{
P Kelly*, , F Paulin', D Lamont ${ }^{2}$, L Baker', S Clearly', D Exon' and A Thompson ',3 \\ 'Dundee Cancer Centre, University of Dundee, Ninewells Hospital and Medical School, Dundee DDI 9SY, UK; '² Post-genomics and Molecular \\ Interactions Centre, School of Life Sciences, University of Dundee, Dow Street, Dundee, UK; ${ }^{3}$ Department of Surgical Oncology, MD Anderson \\ Cancer Center, I 400 Holcombe Boulevard, Houston, TX 77030, USA
}

BACKGROUND: The incidence of oesophageal adenocarcinoma is increasing worldwide but survival remains poor. Neoadjuvant chemotherapy can improve survival, but prognostic and predictive biomarkers are required. This study built upon preclinical approaches to identify prognostic plasma proteomic markers in oesophageal cancer.

METHODS: Plasma samples collected before and during the treatment of oesophageal cancer and non-cancer controls were analysed by surface-enhanced laser desorption/ionisation time-of-flight (SELDI-TOF) mass spectroscopy (MS). Protein peaks were identified by MS in tryptic digests of purified fractions. Associations between peak intensities obtained in the spectra and clinical endpoints (survival, disease-free survival) were tested by univariate (Fisher's exact test) and multivariate analysis (binary logistic regression). RESULTS: Plasma protein peaks were identified that differed significantly $(P<0.05$, ANOVA) between the oesophageal cancer and control groups at baseline. Three peaks, confirmed as apolipoprotein A-I, serum amyloid A and transthyretin, in baseline (pre-treatment) samples were associated by univariate and multivariate analysis with disease-free survival and overall survival. CONCLUSION: Plasma proteins can be detected prior to treatment for oesophageal cancer that are associated with outcome and merit testing as prognostic and predictive markers of response to guide chemotherapy in oesophageal cancer.

British Journal of Cancer (2012) 1 06, 955-961. doi:I0.1038/bjc.2012.15 www.bjcancer.com

Published online 31 January 2012

(c) 2012 Cancer Research UK

Keywords: oesophageal carcinoma; proteomics; biomarkers; survival; disease-free survival

The incidence of oesophageal cancer, particularly adenocarcinoma in western populations, is increasing worldwide (Botterweck et al, 2000; Park, 2002; Lagergren, 2005) and carries a poor prognosis, even in the minority with resectable disease (Gilbert et al, 2002; Munro, 2004) for whom 5 years survival ranges from $10 \%$ to $35 \%$ (Hulscher et al, 2002; Thompson et al, 2007). Trials of neoadjuvant chemotherapy and neoadjuvant chemoradiotherapy have reported mixed results ranging from no difference in curative resection or overall survival (Kelsen et al, 2007) to improved resection rates and survival (MRC, 2002; Geh et al, 2006). A systematic review of 11 randomised controlled trials demonstrated an increase in overall survival with the use of neoadjuvant chemotherapy, but statistical significance was achieved only after 5 years (Maltaner and Fenlon, 2003). Palliative chemotherapy for advanced oesophageal cancer results in the control of symptoms for $70-80 \%$ of patients with $40-50 \%$ objective response rates but only $30-40 \%$ surviving for 1 year (Gilbert et al, 2002).

The ability to identify prognostic biomarkers in oesophageal cancer and to determine, at an early stage, patient prognosis and/ or which patients are most likely to respond to chemotherapy could prevent patients undergoing ineffective and potentially toxic treatments and allow direction of curative or palliative treatment

*Correspondence: Dr P Kelly, E-mail: peter.kelly@axis-shield.com Received 13 October 201।; revised 9 January 2012; accepted 10 January 2012; published online 31 January 2012 to those most likely to benefit. Imaging techniques such as computerised tomography, magnetic resonance imaging, endoscopic ultrasound or positron emission tomography (PET) range in their effectiveness to predict response to chemotherapy (Westerterp et al, 2005). For example, a recent survey of the use of FDG-PET in the prediction of response to neoadjuvant chemotherapy found considerable variability in performance and concluded that the technique should not yet be used in clinical practice to guide therapy decisions (Kwee, 2010). Pathological criteria for assessment of the degree of tumour regression in the resected oesophagus using tumour regression grades may be a significant predictor of disease-free survival (Mandard et al, 1994) but is not an independent prognostic indicator for oesophageal adenocarcinomas (Dunne et al, 2001). Pathological response using modified staging criteria has been shown to predict survival following chemoradiotherapy (Swisher et al, 2005). In addition, pathological response to preoperative chemotherapy has been shown to improve overall survival (Kelsen et al, 2007). However, neither imaging techniques nor resectional pathology have to date provided robust guidance of prognosis or potential response before or during chemotherapy.

There has been growing interest in the use of proteomic methods on peripheral blood plasma to rapidly profile protein markers which link expression of the genome with disease processes (Cahill, 2001; Plebani, 2005) and to discover novel biomarkers of therapeutic response (Smith et al, 2006). Liquid chromatography methods such as high performance liquid 
chromatography and two-dimensional liquid chromatography have been increasingly employed for protein separation and mass spectroscopy (MS) techniques such as matrix-assisted laser desorption ionisation time-of-flight (MALDI-TOF) MS used to analyse the proteins (Colantonio and Chan, 2005). The technique of surface-enhanced laser desorption/ionisation time-of-flight (SELDITOF) MS, in which chromatographic separation is achieved on a solid surface and the proteins analysed intact (Colantonio and Chan, 2005), has been investigated to identify serum and tissue proteomic profiles that could be used in clinical practice.

One study using SELDI-TOF MS reported four peaks of undisclosed identity that differentiated between responders and non-responders in 14 patients treated with chemoradiotherapy for oesophageal squamous cell carcinoma (Hayashida et al, 2005) and were superior to radiological and pathological techniques in assessing response to therapy (Ota et al, 2007), but no comparable studies have been performed on the now more common adenocarcinoma of the oesophagus.

We have previously reported preclinical data from plasma proteomic profiling in human oesophageal cancer (murine) xenografts (Kelly et al, 2010). Comparison of treated and untreated xenograft animals, identified plasma proteins associated with treatment. The purpose of the current study was to develop the preclinical findings and test for clinical associations of plasma proteomic profiling in oesophageal cancer patients before treatment. We sought to test the hypothesis that the expression of circulating proteins, detected by plasma proteomic profiling, may provide clinically useful information about the outcomes for patients with oesophageal carcinoma.

\section{MATERIALS AND METHODS}

\section{Collection of patient samples}

The study 'Diagnostic Markers for the Detection and Monitoring of Oesophageal Disease' was approved by the Tayside Research Ethics Board. Patients were consented on attendance for endoscopy and assigned to the normal or tumour arms of the study (Figure 1), according to the predefined inclusion and exclusion criteria such that the normal patients had no evidence of reflux disease, oesophagitis or cancer and the tumour patients were being assessed for oesophageal cancer before therapy. Baseline heparin plasma samples were collected from all study participants and submitted to the Tayside Tissue Bank for storage at $-70{ }^{\circ} \mathrm{C}$ or below.

Data for each patient included dates of diagnosis, chemotherapy, surgery, recurrence, follow-ups and death (as applicable), allowing a determination to be made of survival and disease-free survival, details of chemotherapy and/or radiotherapy given, pathological response (following surgery) expressed as tumour regression grade according to the Mandard criteria (Mandard et al, 1994), tumour type and staging.

\section{Statistical considerations}

To perform power calculations, data obtained in samples from five patients before and after chemotherapy (Kelly et al, 2010) was analysed. A total of 21 peaks were identified that differed significantly (paired $t$-test) before and after chemotherapy. Using the mean and s.d. data for each of these 21 peaks, it was determined that 20 samples would be sufficient to detect differences between these sample groups for 17 out of $21(81 \%)$ of the peaks with a power of $70 \%$, while 25 samples would be sufficient to detect 17 out of 21 peaks $(81 \%)$ with a power of $80 \%$ and 14 out of 21 peaks (67\%) with a power of $90 \%$. These calculations are in line with the information obtained previously from the literature (Liu and Hwang, 2007). A target for recruitment was therefore 25 samples, recognising analysis on 20 patients, allowing for any necessary exclusions, could be undertaken. Sample statistics were generated to test for significant differences in peak intensities between patient groups (ANOVA).

The relationship between peak intensities, survival status and disease-free status was further analysed at various time points by univariate analysis using two-sided Fisher's exact test with Lancaster's mid-P correction (2FET) and the independent association of each variable further analysed by multivariate analysis using binary logistic regression (BLR). The statistical package used for all analyses including generation of contingency tables was MOSAIC, an internally developed statistical analysis system (implemented in Matlab, Mathworks Inc., Cambridge, UK; Version 6.5 Release 13) For all analyses the null hypothesis was rejected at an $\alpha$ level of $5 \%(P<0.05)$.

\section{Analysis of plasma samples by SELDI-TOF MS}

The analysis of samples reported here was performed once all baseline samples had been collected. As such the data presented

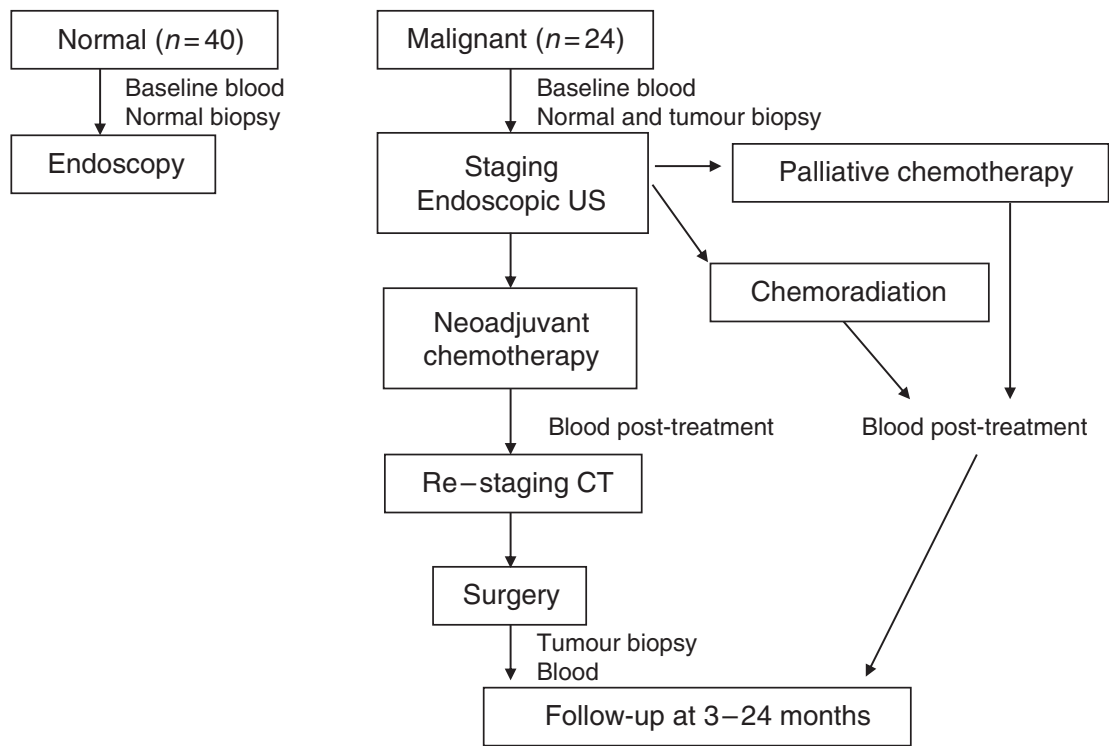

Figure I CONSORT (Schulz et al, 20I0) diagram for the oesophageal cancer clinical study protocol. CT, computed tomography; US, ultrasound. 
here represent an interim analysis in which not all patients were followed up for a full 2-year. All samples were analysed using a single lot of protein chips and buffers over a 3-day period. In order to minimise any differences being observed resulting from analytical effects, the samples were randomised using a random number generator (Excel spreadsheet) to determine the run order. Haemolysed samples were excluded from the analysis.

Samples from each individual were denatured in $9 \mathrm{M}$ urea and tested at a final dilution of $1: 100$ on CM10 (weak cation exchanger) protein chips pre-equilibrated with $50 \mathrm{~mm}$ citrate, $\mathrm{pH}$ 4 and Q10 (strong anion exchanger) protein chips pre-equilibrated with $50 \mathrm{~mm}$ phosphate, $\mathrm{pH} 6$. The chips were washed and sinapinic acid was added as matrix according to the manufacturer's recommended methods (Bio-Rad, Hemel Hempstead, UK), analysed using a SELDI-TOF MS PSII instrument (Bio-Rad) with an all-in-one protein standard (Ciphergen, Fremont, CA, USA) included on each run. Spectra for each individual were collected over a low molecular weight range (2000-30000) and high molecular weight range (20000-150000) using fixed (optimised) laser intensity and detector sensitivity settings. Data collected from multiple points on each spot was collated into averaged spectra using the instrument software. The spectra from each experiment were normalised using the total ion current and calibrated from the all-in-one protein standard data. Peaks were detected using the instrument's biomarker software, using a peak threshold of $20 \%$, a first pass signal/noise ratio of 5 and a second pass signal/noise ratio of 3. Sample statistics were generated for peaks identified using the biomarker software as described above. The performance of this test method was as previously described (Kelly et al, 2010).

\section{Protein identification}

Protein peaks were identified by fractionation followed by MS/MS. Samples containing high-intensity peaks to be identified were fractionated using an off-gel fractionator fitted with 24-cm IPG strips according to the manufacturer's instructions (Agilent Technologies, Santa Clara, CA, USA). Selected fractions were concentrated and desalted in $10 \mathrm{~mm}$ HEPES using molecular weight cut-off filters (Vivaspin, Sartorius, Goettingen, Germany). The retentates were tested by SELDI MS using CM10, Q10, H50 and/or NP20 protein chips to confirm the optimal location of the peak of interest. The desalted fractions were reduced in $10 \mathrm{~mm}$ DTT $(10 \mathrm{~min}$ incubation at $70^{\circ} \mathrm{C}$ ), alkylated in $50 \mathrm{~mm}$ iodoacetamide $(30 \mathrm{~min}$ incubation at room temperature) and loaded onto nu-PAGE $10-20 \%$ Tricine, $10 \%$ or $12 \%$ Bis-Tris gels (Invitrogen, Carlsbad, CA, USA) with MES running buffer and run with SeeBlue Plus 2 prestained standards (Invitrogen). Electrophoresis was performed at $125 \mathrm{~V}$ for $70-90 \mathrm{~min}$ (tricine gels) or $200 \mathrm{~V}$ for $35-40 \mathrm{~min}$ (BisTris gels). Gels were fixed with $50 \%$ methanol/10\% acetic acid and staining was performed with Coomassie G-250 colloidal stain (Invitrogen) for $3-12 \mathrm{~h}$ and destained with deionised water overnight. Selected protein bands were excised from gels using a Harris Unicore 1-mm cutter (Sigma-Aldrich, Dorset, UK). Proteins were passively eluted from the gel pieces to confirm the presence of the peaks of interest by SELDI MS using CM10, Q10, H50 and/or NP20 protein chips. Matched gel pieces were processed and in-gel digested with Trypsin (Roche, Welwyn Garden City, UK) and an aliquot of the digest analysed by nLC-MS-MS using a 4000 QTRAP (Applied Biosystems, Carlsbad, CA, USA). The tandem MS data generated from the observed peptides were analysed and identified using the Mascot search engine (http://www.matrixscience.com) against the IPI human database. Only peptides that had ion scores above the significance threshold were reported and grouped into their respective protein identifications, with MOWSE scores indicating probability of correct identification. Peptide sequences obtained were mapped on the known protein sequence to determine the percentage of the sequence covered.

\section{RESULTS}

In all, 24 subjects with oesophageal cancer and 40 non-cancer patients were enrolled in the study. Among the 24 oesophageal cancer patients (Table 1), 20 had adenocarcinomas, 1 squamous

Table I Summary of oesophageal cancer patients

\begin{tabular}{|c|c|c|c|c|c|c|c|c|}
\hline $\begin{array}{l}\text { Study } \\
\text { number }\end{array}$ & $\begin{array}{c}\text { Age at } \\
\text { diagnosis } \\
\text { (years) }\end{array}$ & Gender & $\begin{array}{l}\text { Ethnic } \\
\text { origin }\end{array}$ & Tumour type & Chemotherapy & $\begin{array}{l}\text { Drug } \\
\text { regime }\end{array}$ & $\begin{array}{l}\text { Further } \\
\text { treatment }\end{array}$ & $\begin{array}{l}\text { Tumour } \\
\text { regression } \\
\text { grade }\end{array}$ \\
\hline T00। & 66 & Male & White & Adenocarcinoma & Neoadjuvant & CF & Surgery & TRG5 \\
\hline T002 & 58 & Male & White & Adenocarcinoma & Neoadjuvant & CF & Surgery & TRG5 \\
\hline T003 & 62 & Male & White & Adenocarcinoma & Neoadjuvant & CF & Surgery & TRG5 \\
\hline T004 & 62 & Male & White & Adenocarcinoma & Neoadjuvant & CF & Surgery & TRG4 \\
\hline T005 & 57 & Male & White & Adenocarcinoma & Neoadjuvant & ECF & Surgery & TRG5 \\
\hline T006 & 58 & Male & White & Adenocarcinoma & Neoadjuvant & CF & Surgery & TRG4 \\
\hline T007 & 51 & Male & White & Adenocarcinoma & Neoadjuvant & CF & Surgery & TRG4 \\
\hline T008 & 58 & Male & White & Adenocarcinoma & Chemoradiation & CF & Stent & N/A \\
\hline T009 & 72 & Male & White & Squamous cell carcinoma & Palliative & ECF & Stent & $N / A$ \\
\hline TOIO & 68 & Male & White & Poorly differentiated carcinoma & Palliative & ECF & None & N/A \\
\hline TOI। & 49 & Male & White & Poorly differentiated carcinoma & Palliative & ECF & None & N/A \\
\hline T012 & 52 & Male & White & Adenocarcinoma & Palliative & ECF & Stent/radiation & N/A \\
\hline T013 & 60 & Male & White & Dysplasia & None & None & None & $N / A$ \\
\hline T014 & 45 & Female & White & Adenocarcinoma & Palliative & ECF & Radiation & N/A \\
\hline T015 & 60 & Male & White & Adenocarcinoma & Neoadjuvant & CF & Surgery\& radiation & TRG5 \\
\hline T016 & 54 & Male & White & Adenocarcinoma & Neoadjuvant & ECF & Surgery & TRG5 \\
\hline T017 & 68 & Male & White & Adenocarcinoma & None & None & Radiation & N/A \\
\hline T0। 8 & 64 & Male & White & Adenocarcinoma & None & None & None & $N / A$ \\
\hline T019 & 51 & Male & White & Adenocarcinoma & Palliative & ECF & None & N/A \\
\hline T020 & 74 & Female & White & Adenocarcinoma & Palliative & $\mathrm{ECaF}$ & None & N/A \\
\hline T02। & 49 & Male & White & Adenocarcinoma & Neoadjuvant & CF & Surgery & TRG4 \\
\hline T022 & 71 & Female & White & Adenocarcinoma & Palliative & MF & None & N/A \\
\hline T023 & 58 & Female & White & Adenocarcinoma & Neoadjuvant & CF & Surgery & TRG5 \\
\hline T024 & 64 & Male & White & Adenocarcinoma & Chemoradiation & $\mathrm{F}$ & Radiation & N/A \\
\hline
\end{tabular}

Abbreviations: $C=$ cisplatin; $C a=$ carboplatin; $E=$ epirubicin; $F=5$-fluorouracil; $M=$ mitomycin $c$. 
Table 2 Summary of case controls

\begin{tabular}{|c|c|c|c|c|}
\hline $\begin{array}{l}\text { Study } \\
\text { number }\end{array}$ & $\begin{array}{l}\text { Age at } \\
\text { recruitment } \\
\text { (years) }\end{array}$ & Gender & $\begin{array}{l}\text { Ethnic } \\
\text { origin }\end{array}$ & Indication for endoscopy \\
\hline NOOI & 54 & Female & White & Microcytic anaemia \\
\hline N002 & 26 & Male & White & Coeliac screening \\
\hline N003 & 23 & Female & White & Anaemia \\
\hline N004 & 53 & Female & White & Anaemia \\
\hline N005 & 54 & Female & White & Anaemia \\
\hline N006 & 46 & Female & White & Diarrhoea \\
\hline N007 & 74 & Male & White & Anaemia \\
\hline N008 & 34 & Male & White & Dyspepsia \\
\hline N009 & 44 & Female & White & Epigastric pain \\
\hline NOIO & 63 & Female & White & Epigastric pain \\
\hline NOII & 77 & Female & White & Dyspepsia, dilated pancreatic duct \\
\hline $\mathrm{NOI} 2$ & 53 & Male & White & Epigastric Pain \\
\hline N0I3 & 69 & Female & White & Dyspepsia \\
\hline Nol4 & 59 & Female & White & Dyspepsia, previous salmonella \\
\hline Nol5 & 70 & Male & White & Maleana \\
\hline Nol6 & 36 & Female & White & Abdominal pain \\
\hline Nol7 & 46 & Female & White & Dyspepsia \\
\hline N0I8 & 58 & Female & White & Microcytic anaemia \\
\hline Nol9 & 41 & Male & White & Epigastric Pain \\
\hline N020 & 58 & Male & White & Nausea and vomiting \\
\hline N02I & 24 & Female & White & Coeliac screening \\
\hline N022 & 60 & Female & White & Iron deficiency anaemia \\
\hline N023 & 57 & Female & White & Microcytic anaemia \\
\hline N024 & 39 & Male & White & Dyspepsia \\
\hline N025 & 64 & Male & White & Dyspepsia \\
\hline N026 & 59 & Female & White & Iron deficiency anaemia \\
\hline N027 & 73 & Female & White & Altered bowel habit and anaemia \\
\hline N028 & 46 & Female & White & Anaemia \\
\hline N029 & 49 & Female & White & Abdominal pain and weight loss \\
\hline N030 & 58 & Female & White & Weight loss \\
\hline N03I & 79 & Male & White & Anaemia \\
\hline N032 & 69 & Male & White & Anaemia \\
\hline N033 & 23 & Female & White & Dyspepsia \\
\hline N034 & 24 & Male & Asian & Weight loss \\
\hline N035 & 54 & Female & White & Dyspepsia \\
\hline N036 & 71 & Female & White & Weight loss \\
\hline N037 & 65 & Female & White & Dyspepsia and weight loss \\
\hline N038 & 56 & Female & White & Weight loss and vomiting \\
\hline N039 & 32 & Male & White & Abdominal pain and possible maleana \\
\hline N040 & 63 & Female & White & Weight loss \\
\hline
\end{tabular}

cell carcinoma, 2 poorly differentiated carcinomas and 1 severe dysplasia. Eleven (all adenocarcinoma) patients received neoadjuvant chemotherapy followed by surgery, eight patients received palliative chemotherapy and two received chemoradiation. For the 11 adenocarcinoma patients who received neoadjuvant chemotherapy, the pathological response (tumour regression grade, TRG) was scored as TRG4 (residual cancer outgrowing fibrosis) in 4 cases and TRG5 (absence of regressive change) in 7 cases (Mandard et al, 1994). At the time of this interim analysis all surviving patients had been followed up for at least 12 months. Overall survival in the 24 oesophageal cancer patients ranged from 2 months to $>24$ months from the date of diagnosis, median survival was 16.9 months (median survival 17.5 months). Of the 13 patients who had died to date at the time of analysis, mean survival was 8.7 months (median 9.3 months). Survival in the neoadjuvant chemotherapy group ranged from 8 months to greater than 12 months. Given the survival data, it was deemed sufficient data had been generated to perform the analysis at this stage rather than waiting for all surviving patients to be followed up to 24 months.

The 40 non-cancer patients (Table 2) had a mean age of 52.5 years (median 55, range 23-79) and 13 out of $40(32.5 \%)$ were male, whereas the oesophageal cancer group (Table 1) had a mean age of 59.5 (median 59, range $45-74$ ) and 20 out of $24(83.3 \%$ ) were male. Hence, although any differences observed between the two groups are unlikely to be affected by the similar age ranges, differences due to gender might be observed. All but one case control and all the oesophageal cancer cases were Caucasian.

SELDI-TOF MS spectra were obtained in plasma samples from the normal and malignant arms of the study at baseline, together with the plasma samples collected at specified time points (Figure 1). The spectra were analysed to detect statistically significant differences (ANOVA $P<0.05$ ) in peak intensities between the sample groups. Peaks that differed significantly at baseline between the non-cancer patients $(n=36)$ and oesophageal cancer patients $(n=21)$ are shown in Figure 2. Peaks that differed significantly between normal men and women were excluded from this analysis to correct for any gender bias between the normal and malignant groups. Protein peaks were detected that differed significantly at baseline between a group of five patients who survived for $>11$ months (good survivors) and a group of five
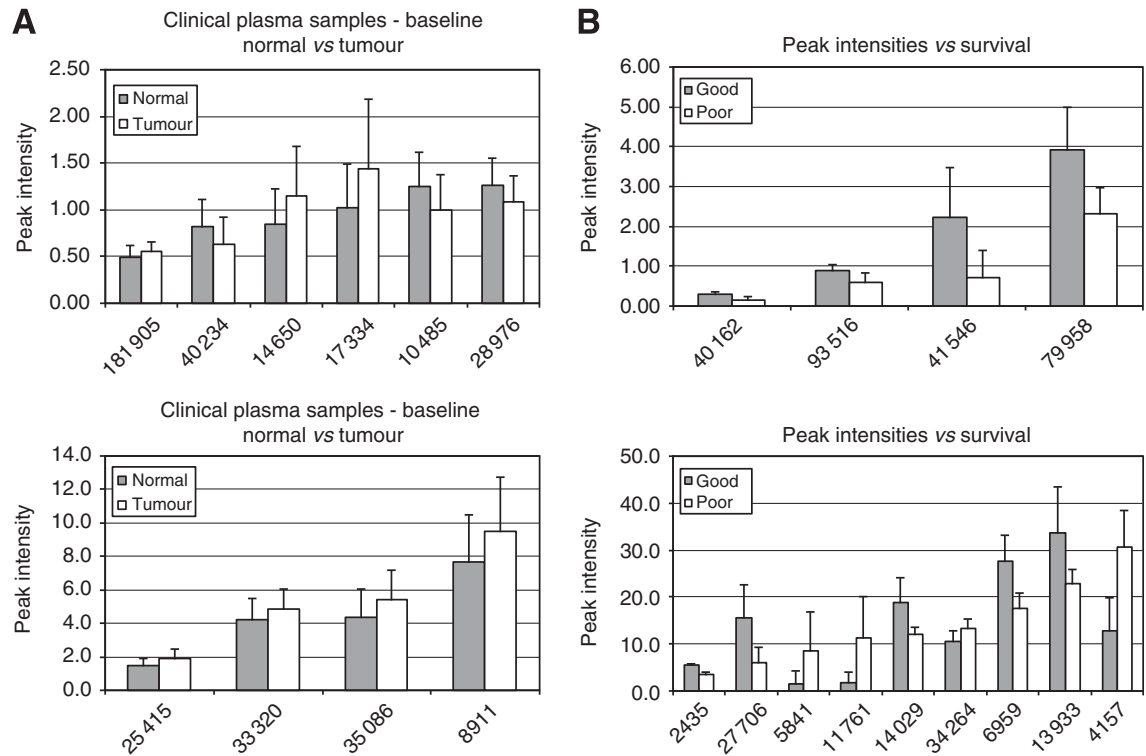

Figure 2 Peak intensity data at baseline for $(\mathbf{A})$ oesophageal cancer patients vs case controls and (B) according to survival. Sample group statistics (mean and s.d.) obtained for $(\mathbf{A})$ plasma peaks $(\mathrm{m} / \mathrm{z})$ differing significantly $(P<0.05$, appropriate parametric or non-parametric test) between case controls (normal, $n=36$ ) and oesophageal cancer (tumour, $n=2 I$ ) patients at baseline or $(\mathbf{B})$ according to survival from date of diagnosis, between the greater than II months group (good, $n=5$ ) and the $<7$ months group (poor, $n=5$ ). 
patients who survived for $<7$ months (poor survivors, Figure 2B) or between good and poor disease-free survivors (Figure 3 ).

To further test the hypothesis that markers for survival and disease-free survival had been detected, statistical analysis was performed on the whole oesophageal cancer cohort after exclusion of the hyperplasia patient and three haemolysed samples (20 patients remaining). Peak intensity data generated using this dataset were assessed using histograms and dot plots to determine a cut-off and the results for each sample scored as high or low (above or below the cut-off). The relationship between a high or
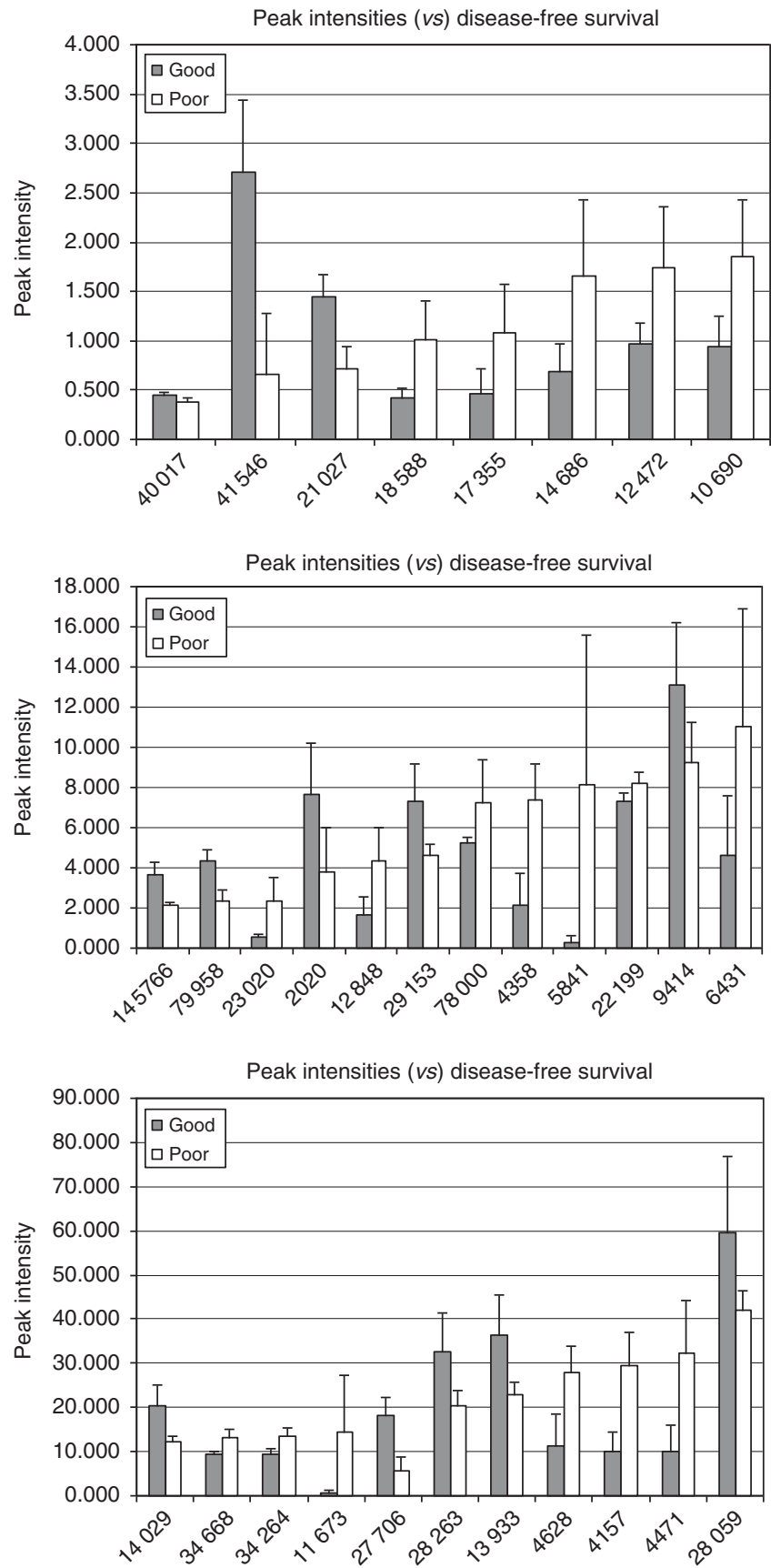

Figure 3 Peak intensity data at baseline according to disease-free survival. Sample group statistics (mean and s.d.) obtained for plasma peaks $(\mathrm{m} / \mathrm{z})$ differing significantly $(P<0.05$ appropriate parametric or nonparametric test), according to disease-free survival from date of diagnosis, between the $>$ II months group (good, $n=4$ ) and the $<7$ months group (poor, $n=6$ ). low baseline result and survival or disease-free survival was tested by univariate analysis using Fisher's exact test (with Lancaster's mid-point correction) and confirmed an association $(P<0.05)$ between peak intensity and survival or disease-free survival (by month) for several peaks at three or more time points.

To further test the associations detected by univariate analysis, multivariate analysis was performed (BLR). From the univariate analysis, five peaks were identified that gave identical responses (all five were high in the same patients and all five were low in the same patients). These were peaks at $(\mathrm{m} / z) 27706$ and as well as peaks at $(\mathrm{m} / \mathrm{z}) 34264,41546$ and 79958. In addition, a peak at $(\mathrm{m} / \mathrm{z}) 23020$ gave an exactly opposite response to these five markers. It was necessary to exclude all but one of these $(\mathrm{m} / z 79958$ retained) in order to perform multivariate analysis. Results obtained for the $(\mathrm{m} / z) 79958$ peak apply equally to the other four peaks and inversely for $(\mathrm{m} / \mathrm{z}) 23020$. A further two peaks at $(\mathrm{m} / \mathrm{z}) 5841$ and 11670 (both from the low molecular weight analysis of the CM10 chip) gave nearly identical results to the peak at $(\mathrm{m} / \mathrm{z}) 23020$ and were similarly excluded.

Multivariate analysis was performed on all remaining peaks, to test for associations with disease-free survival at months 4 and 8 , and survival at months 8 and 12. These time points were selected as optimal in terms of their bimodal discrimination for clinical survival in the cancer study population, consistent with probability values obtained for all the markers by Fisher's exact test.

Two peaks at $(\mathrm{m} / \mathrm{z}) 79958$ and 34668 were found to be significantly associated $(P<0.05)$ with each other and with survival at 8 and 12 months. The peak at $(\mathrm{m} / \mathrm{z}) 79958$ was independently associated with survival, whereas the peak at $(\mathrm{m} / z) 34668$ was not. The same two peaks were both found to be independently associated with disease-free survival at 4 months. In addition, the peak at 14029 was found to be independently associated with disease-free survival at 8 months. By their identical responses, the findings for the peak at $(\mathrm{m} / \mathrm{z}) 79958$, also apply to the peaks at $(\mathrm{m} / z) 27765$ (or 27 706), 34264 and 41546 , and apply negatively to the peaks at $(\mathrm{m} / \mathrm{z}) 23020,11670$ and 5841 (Figure 4).

Two groups of markers were detected that were associated with each other:

(1) Peaks at $(\mathrm{m} / \mathrm{z}) 12848,14686,10690$ and 18588. None of these were associated with survival or disease-free survival.

(2) Peaks at $(\mathrm{m} / \mathrm{z}) 4471,28060,28263,13933,14029$ and 4157. This grouping includes the peak at $(\mathrm{m} / z) 14029$ that was independently associated with disease-free survival at 8 months.

From peptide fingerprinting analysis of fractionated samples, three of the protein peaks that were independently associated with survival and/or disease-free survival were identified at the appropriate expected molecular weights as serum amyloid A $(\mathrm{m} / z \mathrm{11} 670)$, transthyretin $(\mathrm{m} / z \mathrm{14} 029)$ and apolipoprotein A-I $(\mathrm{m} / z 27665)$ with high Mowse probability scores (1131 or greater), multiple peptides detected (37 or more) and high sequence coverage ( $42 \%$ or higher).

\section{DISCUSSION}

This proteomic profiling study sought pre-treatment plasma proteins associated with better disease-free or overall survival following therapy for oesophageal cancer, informed by preclinical findings from a murine oesophageal cancer xenograft model.

Significant differences were observed in baseline plasma samples between the non-cancer and oesophageal cancer groups for a total of 10 protein peaks.

Univariate analysis demonstrated associations between the baseline peak intensity data for 9 protein peaks significant for survival and 14 peaks significant for disease-free survival at multiple time points (1-12 months following diagnosis). 

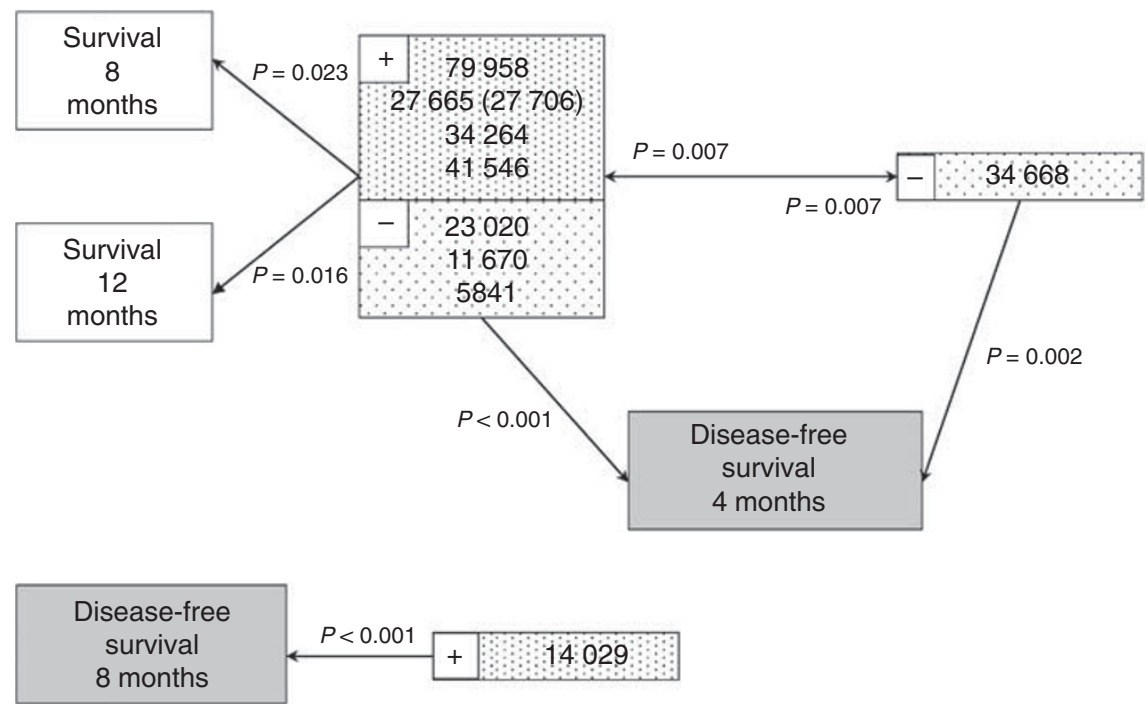

Figure 4 Multivariate analysis, associations with survival and disease-free survival. Associations between peaks $(\mathrm{m} / \mathrm{z})$ intensities and survival or disease-free survival determined by BLR. Direct lines indicate statistically significant independent associations ( $P$-values shown). Positive $(+)$ and negative $(-)$ associations are depicted.

Multivariate analysis demonstrated that four of the peaks $(\mathrm{m} / \mathrm{z}$ $79958,27665,34264$ and 41546$)$ were positively associated and three peaks $(\mathrm{m} / \mathrm{z} 23020,11670$ and 5841$)$ negatively associated independently with survival at 8 and 12 months. These peaks and a further peak $(\mathrm{m} / \mathrm{z} 34668)$ were also independently associated with disease-free survival at 4 months, and a further peak $(\mathrm{m} / z$ 14029) was independently associated with disease-free survival at 8 months.

Included among the peaks that were associated with survival or disease-free survival, both by univariate analysis and multivariate analysis are three peaks identified as SAA $(\mathrm{m} / \mathrm{z} 11670)$, transthyretin $(m / z 14029)$ and apolipoprotein A-I $(m / z 27665)$. We previously reported that these three proteins were associated with response to chemotherapy treatment in a mouse xenograft model (Kelly et al, 2010). The present study therefore confirms that these three plasma proteins are of interest in patients with oesophageal cancer and are associated with disease-free survival or overall survival. Also included among these peaks were another five peaks $(\mathrm{m} / \mathrm{z} 79958,34264,41546,23030$ and 5841), not predicted by the murine work, the identities of which are unknown.

The three peaks for which proteins were identified, Serum Amyloid A, transthyretin and apolipoprotein A-I are consistent with data from a variety of other cancers. As markers of inflammation and acute phase responses (Malmendier et al, 1988; Raynes et al, 1991; Juan et al, 2004; Malle et al, 2009) they may be non-specific markers for oesophageal cancer as individual proteins, confounded by inflammatory processes that occur in other disease states (Chechlinska et al, 2010). However, their detection may result from the known involvement of inflammatory mechanisms in the development of oesophageal cancer (AbdelLatif et al, 2009). Indeed there is now good evidence that markers of systemic inflammatory response are prognostic cancer biomarkers, particularly in colorectal, gastro-oesophageal and renal cancers (Roxburgh and McMillan, 2010), and a prognostic score based on serum c-reactive protein and albumin (the Glasgow Prognostic Score) has been proposed for oesophageal cancer (Vashist et al, 2011). Therefore, the combination of changes observed in plasma for the markers observed in the present study merits testing as a prognostic signature for oesophageal cancer, a hypothesis that would require further validation.
A similar study performed by SELDI-TOF MS in patients with oesophageal squamous cell carcinomas proposed four unidentified serum markers $(\mathrm{m} / \mathrm{z} 7420,9112,17123$ and 12867$)$ associated with chemoradiotherapy response in 14 out of 15 patients (Hayashida et $a l, 2005)$ as the only significant association with outcome by multivariate analysis (Ota et al, 2007). The current study, performed with plasma samples predominantly in patients with adenocarcinomas, did not confirm this previously reported serum panel. One peak with a very similar $\mathrm{m} / \mathrm{z}$ ratio of 12848 was detected but not found to be associated with survival or diseasefree survival. The different markers detected in these two studies may reflect differences resulting from sample collection (the use of serum $v s$ plasma) and processing, including differences in selection of chip surfaces and/or buffer conditions, or differences in the patient population (both geographic and squamous cell $v s$ adenocarcinoma), reflecting the differences seen in the preclinical models of OE21 (squamous cell carcinoma) and OE19 (adenocarcinoma) treatment markers (Kelly et al, 2010). Alternatively such differences may simply reflect the small population sizes in the two studies pointing to the need for further validation cohorts.

Much has been made in the literature (Kerr and Midgley, 2010) about the plethora of candidate markers that have been proposed in the cancer field, particularly in the case of proteomics studies where underpowered studies have resulted in marker profiles which cannot subsequently be validated (McLerran et al, 2008). The clinical study described here has extended preclinical xenograft data (Kelly et al, 2010) and demonstrated associations between baseline (pre-treatment) plasma proteins and oesophageal cancer survival. The variety of therapeutic approaches, small cohort size and poor responses to therapy do not allow for testing the predictive value of the plasma markers in response to therapy, but warrant further clinical investigation involving a larger patient cohort, both to independently verify the prognostic findings and to further elucidate the predictive clinical utility of these markers.

\section{ACKNOWLEDGEMENTS}

We would like to acknowledge the help received from the Tayside Tissue bank and funding provided by Axis-Shield Ltd. for this study. 


\section{REFERENCES}

Abdel-Latif MM, Duggan S, Reynolds JV, Kelleher D (2009) Inflammation and esophageal carcinogenesis. Curr Opin Pharmacol 9: 396-404

Botterweck AA, Schouten LJ, Volovics A, Dorant E, van Den Brandt PA (2000) Trends in incidence of adenocarcinoma of the oesophagus and gastric cardia in ten European countries. Int J Epidemiol 29: 645-654

Cahill DJ (2001) Protein and antibody arrays and their medical applications. J Immunol Methods 250: 81 -91

Chechlinska M, Kowalewska M, Nowak R (2010) Systemic inflammation as a confounding factor in cancer biomarker discovery and validation. Nat Rev Cancer 10: 2-3

Colantonio DA, Chan DW (2005) The clinical application of proteomics. Clin Chim Acta 357: $151-158$

Dunne B, Reynolds JV, Mulligan E, Kelly A, Griffin M (2001) A pathological study of tumour regression in oesophageal adenocarcinoma treated with preoperative chemoradiotherapy. J Clin Pathol 54: $841-845$

Geh JI, Bond SJ, Bentzen SM, Glynne-Jones R (2006) Systematic overview of preoperative (neoadjuvant) chemoradiotherapy trials in oesophageal cancer: evidence of a radiation and chemotherapy dose response. Radiother Oncol 78: 236-244

Gilbert FJ, Park KGM, Thompson AM (2002) Scottish Audit of Gastric and Oesophageal Cancer. Report 1997-2000. Scottish Audit of Gastric and Oesophageal Cancer Steering Group: Edinburgh http://www.crag.scot.nhs.uk/committees/ceps/reports/0_prelims.pdf

Hayashida Y, Honda K, Osaka Y, Hara T, Umaki T, Tsuchida A, Aoki T, Hirohashi S, Yamada T (2005) Possible prediction of chemoradiosensitivity of esophageal cancer by serum protein profiling. Clin Cancer Res 11: $8042-8047$

Hulscher JB, van Sandick JW, de Boer AG, Wijnhoven BP, Tijssen JG, Fockens P, Stalmeier PF, ten Kate FJ, van Dekken H, Obertop $\mathrm{H}$, Tilanus HW, van Lanschot JJ (2002) Extended transthoracic resection compared with limited transhiatal resection for adenocarcinoma of the esophagus. $N$ Engl J Med 347: $1662-1669$

Juan HF, Chen JH, Hsu WT, Huang SC, Chen ST, Yi-Chung Lin J, Chang YW, Chiang CY, Wen LL, Chan DC, Liu YC, Chen YJ (2004) Identification of tumor-associated plasma biomarkers using proteomic techniques: from mouse to human. Proteomics 4: 2766-2775

Kelly P, Appleyard V, Murray K, Paulin F, Lamont D, Baker L, Suttie S, Exon D, Thompson A (2010) Detection of oesophageal cancer biomarkers by plasma proteomic profiling of human cell line xenografts in response to chemotherapy. Br J Cancer 103: 232-238

Kelsen DP, Winter KA, Gunderson LL, Mortimer J, Estes NC, Haller DG, Ajani JA, Kocha W, Minsky BD, Roth JA, Willett CG (2007) Long-term results of RTOG trial 8911 (USA Intergroup 113): a random assignment trial comparison of chemotherapy followed by surgery compared with surgery alone for esophageal cancer. J Clin Oncol 25: 3719-3725

Kerr DJ, Midgley R (2010) Defective mismatch repair in colon cancer: a prognostic or predictive biomarker? J Clin Oncol 28: 3210-3212

Kwee RM (2010) Prediction of tumor response to neoadjuvant therapy in patients with esophageal cancer with use of 18F FDG PET: a systematic review. Radiology 254: $707-717$

Lagergren J (2005) Adenocarcinoma of oesophagus: what exactly is the size of the problem and who is at risk? Gut 54(Suppl 1): i1 -i5

Liu P, Hwang JT (2007) Quick calculation for sample size while controlling false discovery rate with application to microarray analysis. Bioinformatics 23: $739-746$

Malle E, Sodin-Semrl S, Kovacevic A (2009) Serum amyloid A: an acute-phase protein involved in tumour pathogenesis. Cell Mol Life Sci 66: $9-26$

Malmendier CL, Lontie JF, Sculier JP, Dubois DY (1988) Modifications of plasma lipids, lipoproteins and apolipoproteins in advanced cancer patients treated with recombinant interleukin-2 and autologous lymphokine-activated killer cells. Atherosclerosis 73: 173-180

Maltaner R, Fenlon D. Preoperative chemotherapy for resectable thoracic oesophageal cancer. Cochrane Database Syst Rev 2003 CD001556

Mandard AM, Dalibard F, Mandard JC, Marnay J, Henry-Amar M, Petiot JF, Roussel A, Jacob JH, Segol P, Samama G, Ollivier J-M, Bonvalot MD, Gignoux M (1994) Pathologic assessment of tumor regression after preoperative chemoradiotherapy of esophageal carcinoma. Clinicopathologic correlations. Cancer 73: 2680-2686

McLerran D, Grizzle WE, Feng Z, Thompson IM, Bigbee WL, Cazares LH, Chan DW, Dahlgren J, Diaz J, Kagan J, Lin DW, Malik G, Oelschlager D, Partin A, Randolph TW, Sokoll L, Srivastava S, Srivastava S, Thornquist M, Troyer D, Wright GL, Zhang Z, Zhu L, Semmes OJ (2008) SELDI-TOF MS whole serum proteomic profiling with IMAC surface does not reliably detect prostate cancer. Clin Chem 54: 53-60

MRC (Oesophageal Working Party) 2002 Surgical resection with or without preoperative chemotherapy in oesophageal cancer: a randomised controlled trial. Lancet 359: 1727 - 1733

Munro AJ (2004) Oesophageal cancer: a view over overviews. Lancet 364: $566-568$

Ota Y, Takagi Y, Osaka Y, Shinohara M, Hoshino S, Tsuchida A, Aoki T, Honda K, Yamada T (2007) Usefulness of serum protein profiling for prediction of preoperative chemoradiosensitivity of esophageal cancer. Oncol Rep 18: $653-657$

Park KG (2002) Gastro-oesophageal cancer: facts, myths and surgical folk lore. J R Coll Surg Edinb 47: 716-730

Plebani M (2005) Proteomics: the next revolution in laboratory medicine? Clin Chim Acta 357: 113-122

Raynes JG, Eagling S, McAdam KP (1991) Acute-phase protein synthesis in human hepatoma cells: differential regulation of serum amyloid A (SAA) and haptoglobin by interleukin-1 and interleukin-6. Clin Exp Immunol 83: $488-491$

Roxburgh CS, McMillan DC (2010) Role of systemic inflammatory response in predicting survival in patients with primary operable cancer. Future Oncol 6: $149-163$

Schulz KF, Altman DG, Moher D (2010) CONSORT 2010 statement: updated guidelines for reporting parallel group randomized trials. Ann Intern Med 152: 726-732

Smith L, Lind MJ, Welham KJ, Cawkwell L (2006) Cancer proteomics and its application to discovery of therapy response markers in human cancer. Cancer 107: 232-241

Swisher SG, Hofstetter W, Wu TT, Correa AM, Ajani JA, Komaki RR, Chirieac L, Hunt KK, Liao Z, Phan A, Rice DC, Vaporciyan AA, Walsh GL, Roth JA (2005) Proposed revision of the esophageal cancer staging system to accommodate pathologic response (pP) following preoperative chemoradiation (CRT). Ann Surg 241: 810-817; discussion $817-820$

Thompson AM, Rapson T, Gilbert FJ, Park KG (2007) Hospital volume does not influence long-term survival of patients undergoing surgery for oesophageal or gastric cancer. Br J Surg 94: 578-584

Vashist YK, Loos J, Dedow J, Tachezy M, Uzunoglu G, Kutup A, Yekebas EF, Izbicki JR (2011) Glasgow Prognostic Score is a predictor of perioperative and long-term outcome in patients with only surgically treated esophageal cancer. Ann Surg Oncol 18: 1130-1138

Westerterp M, van Westreenen HL, Reitsma JB, Hoekstra OS, Stoker J, Fockens P, Jager PL, Van Eck-Smit BL, Plukker JT, van Lanschot JJ, Sloof GW (2005) Esophageal cancer: CT, endoscopic US, and FDG PET for assessment of response to neoadjuvant therapy - systematic review. Radiology 236: $841-851$

This work is published under the standard license to publish agreement. After 12 months the work will become freely available and the license terms will switch to a Creative Commons Attribution-NonCommercial-Share Alike 3.0 Unported License. 